J. Clin. Chem. Clin. Biochem.

Vol. 16, 1978, pp. 191-195

\title{
Kinetische immunologisch-turbidimetrische C3-Bestimmung mit einem Fast Analyzer
}

\author{
Von H. Ebeling \\ Institut für Klinische Chemie und Klinische Biochemie (Direktor: Prof. Dr. H.-J. Dulce) am Klinikum Steglitz \\ der Freien Universität Berlin
}

(Eingegangen am 21. Juli 1977)

Zusammenfassung: Es wird eine neue immunologisch-turbidimetrische Methode zur quantitativen Einzel-ProteinBestimmung vorgestellt. Sie beruht im Prinzip auf einer Initial-Rate-Analyse mit Fixed-Time-Kinetik. Für das Protein-Beispiel C3 werden optimale Bedingungen beschrieben für das Puffermilieu, die Reaktionszeitdauer, die ,InitialRate"-Intervallzeit, die Antigen- und Antikörper-Konzentration. Mit diesen optimierten, standardisierten Bedingungen ist man in der Lage, bei einem Reaktionszeitintervall von $\Delta \mathrm{A} / 15$ Sekunden bereits nach 20 Sekunden Meßergebnisse von 29 Proben zu erhalten. Bei guter relativer Richtigkeit und einem Variationskoeffizienten von Tag zu Tag von 4,3 bzw. 6,4\% für C3-Konzentrationen von 217,5 bzw. $435 \mathrm{mg} / 1$ empfiehlt sich diese Technik für das klinischchemische Laboratorium. An anderer Stelle wird ausführlich über Methodenvergleiche und Messungen von Patientensera berichtet werden.

\section{Kinetic immunological-turbidimetric determination of C-3 protein with a Fast Analyzer}

Summary: A new immunological-turbidimetric method is described for the quantitative determination of individual proteins. It is based on initial rate analysis with fixed time kinetics. The optimal conditions for the analysis of C-3 protein are described, with respect to buffer composition, reaction time, the time interval for measurement of the initial rate, and the concentrations of antigen and antibody. With these optimised, standardised conditions, it is possible to obtain results for 29 samples in 20 seconds, using a reaction time of $\Delta \mathrm{A} / 15$ seconds. The method is relatively accurate with a day to day variation coefficient of 4.3 for C-3 concentrations of $217.5 \mathrm{mg} / 1$, and 6.4 for concentrations of $435 \mathrm{mg} / \mathrm{l}$. It is therefore suitable for the clinical chemical laboratory. A detailed comparison of this with other methods, and its use in the measurement of patients' sera will be presented elsewhere.

\section{Einleịtung und Fragestellung}

Zunehmend setzen sich in der Klinischen Chemie mechanisierte Analysensysteme zum quantitativen, immunologischen Bestimmen von Antigenen und Antikörpern durch. Meist beruhen diese Systeme auf dem Äquilibrium-Meßprinzip (1-3). In Anlehnung an Buffone et al. (4), (5) wird in der vorliegenden Arbeit versucht, mit dem auf dem Andersonschên Prinzip (6) beruhenden Zentrifugen-Anàlysator, CENTRIFICHEM SYSTEM 400, quantitativ, immunoturbidimetrisch Antigene und Antikörper zu bestimmen. Ähnlich wie bei neueren SubstratBestimmungs-Methoden sollte hier das Meßprinzip von „Initial-Rate“- bzw. „Short-Fixed-Time"-Analysen zur C3-Bestimmung herangęogen werden.

\section{Material und Methoden}

Geräte

Zentrifugen-Fast-Analyzer CENTRIFICHEM SYSTEM 400, Fa. UNION CARBIDE/LA ROCHE, Basel.

\section{Reagenzien}

Britton-Robinson-Puffer-NaCl-Lösung (plus $35 \mathrm{mg} / 1$ Netzmittel

Triton X-100), pH 6,00, $11 \mathrm{mmol} / 1$ (2).

Standard-Human-Serum Batch No. $1001 \mathrm{C}$,

Protein-Standard-Serum B Batch No. 475 D,

Human-Plasmaprotein-Antiserum vom Kaninchen Anti- $\beta_{1} C / \beta_{1} A$ -

Globulin Serum Batch No. 5106A,

Fa. BEHRINGWERKE AG, Marburg.

\section{Vorgehen}

Materialgewinnung

Sofort nach venöser Blutentnahme Abfüllen von $2 \mathrm{ml}$ Blut in Spezialgefäße aus Glas, die üblicherweise zum Bestimmen von 
Fibrinogen-Spaltprodukten dienen (an anderer Stelle wird dieses Vorgehen begründet werden). Sample Collection Tubes aus THROMBO-WELLCOTEST, Fa. WELLCOME REAGENTS LIMITED, Beckenham, England, enthalten pro Glasröhrchen folgende Zusätze:

etwa 3600 NF Units Sojabohnen-Trypsin-Inhibitor und 20 NIH Units Thrombin vom Rind.

Anschließend sofortiges gründliches Durchmischen und Ausgerinnenlassen der Blutprobe bei $37^{\circ} \mathrm{C}$ im Brutschrank für etwa 30 Minuten. Dann Zentrifugieren und Abhebern des SerumÜberstandes und Lagern des Probenmaterials bei $-20^{\circ} \mathrm{C} \mathrm{im}$ Gefrierschrank bis zum Bestimmen.

\section{Proben- und Antiserum-Vorbereitung}

Proben-Vorverdünnung z. B. 1:2 und Antiserum-Vorverdünnung z. B. 1:10 mit der Puffer-Lösung (s. o.). Dabei muß darauf geachtet wer, daß die Konzentration der vorverdünnten Probe auf dem aufsı igenden Schenkel der ,Heidelberger-Kurve" zur Antigen-Bestimmung liegen muß (2).

\section{Kalibrierung}

Erstellen der Standardkurve aus Standard-Human-Serum-Vorverdünnungen im C3-Konzentrationsbereich von 0 bis etwa $600 \mathrm{mg} / \mathrm{l}$ in 50 bis $100 \mathrm{mg} / \mathrm{l}$-Schritten.

\section{Volumina-Einstellungen am Pipettor}

sample (= vorverdünnte Probe) $5 \mu 1$

sample + diluent (= vorverdünnte Probe plus Puffer-Lösung) $95 \mu \mathrm{l}$ reagent (= vorverdünntes Antiserum) $250 \mu \mathrm{l}$.

\begin{tabular}{ll}
\multicolumn{2}{l}{ Analyzer-Einstellungen } \\
temperature & $25^{\circ} \mathrm{C}$ \\
filter & $292 \mathrm{~nm}$ \\
$\mathrm{~T}_{\text {o time delay }}$ & $005 \mathrm{sec}$. \\
$\Delta \mathrm{T}$ time interval & $00: 15 \mathrm{~min}$. \\
abnormal absorbance & $4,9 \mathrm{U}$ \\
blank & AUTO \\
test mode & RATE \\
print out & CONC. \\
concentration factor & 1000 \\
no. of prints & 1 \\
test code & 00
\end{tabular}

\section{Berechnung}

1. Graphische Darstellung der Standardkurve:

Ordinate: Trübungsänderung/Zeitintervall $\cong(\Delta A) / \Delta t), z$. B. $\Delta A / 15$ s.

Abszisse: Konzentration in $\mathrm{mg} / \mathrm{l}$.

2. Ermittlung der Probenkonzentration:

Auf der Standardkurve wird für das gemessene $\Delta \mathrm{A} / 15 \mathrm{~s}$ der unbekannten, vorverdünnten Probenkonzentration die dazugehörige Konzentration auf der Abszisse aufgesucht und zur Berechnung der Ausgangskonzentration mit dem Verdünnungsfaktor multipliziert.

\section{Ergebnisse und Diskussion}

Beim Erarbeiten dieser Methode haben wir uns eng an die von uns ermittelten optimierten Bedingungen zur mechanisierten immunologisch-nephelometrischen Antigen- und Antikörper-Bestimmung über den TECHNICON AUTOANALYZER II gehalten (2). Dabei hat sich wiederum die Britton-Robinson-Puffer-NaCl-Lösung, pH 6,00,
$11 \mathrm{mmol} / 1$, gut bewährt im Gegensatz zu anderen Verdünnungsmitteln wie physiologische $\mathrm{NaCl}$-Lösung mit und ohne Polyethylenglykol-Zusatz $(2-5,1)$.

Wie die Abbildung 1 zeigt, sind Reaktionsgeschwindigkeiten zum Zeitpunkt $t_{0}$ nur für relativ niedrigere C3Konzentrationen unter diesen Bedingungen meßbar. Mit zunehmeñder Antigen-Konzentration verschieben sich die Kurvenscheitelpunkte weiter nach links, so daß die Anfangsgeschwindigkeiten hier höher liegen und unsere „Short-Fixed-Time"-Meßwerte bereits auf den absteigenden Schenkeln der Reaktionszeit-Kurven liegen. $\mathrm{Da}$ jedoch die kürzest mögliche Reaktionszeit, gerätebedingt, 15 Sekunden beträgt, konnten nur für C3-Konzentrationen kleiner als $100 \mathrm{mg} / 1$,Initial-Rate"-Kinetiken gemessen werden. Für C3-Konzentrationen größer als $100 \mathrm{mg} / 1$ konnten folglich ,Short-Fixed-Time“" Kinetiken nicht als echte „Initial-Rate“-Analysen durchgeführt werden.

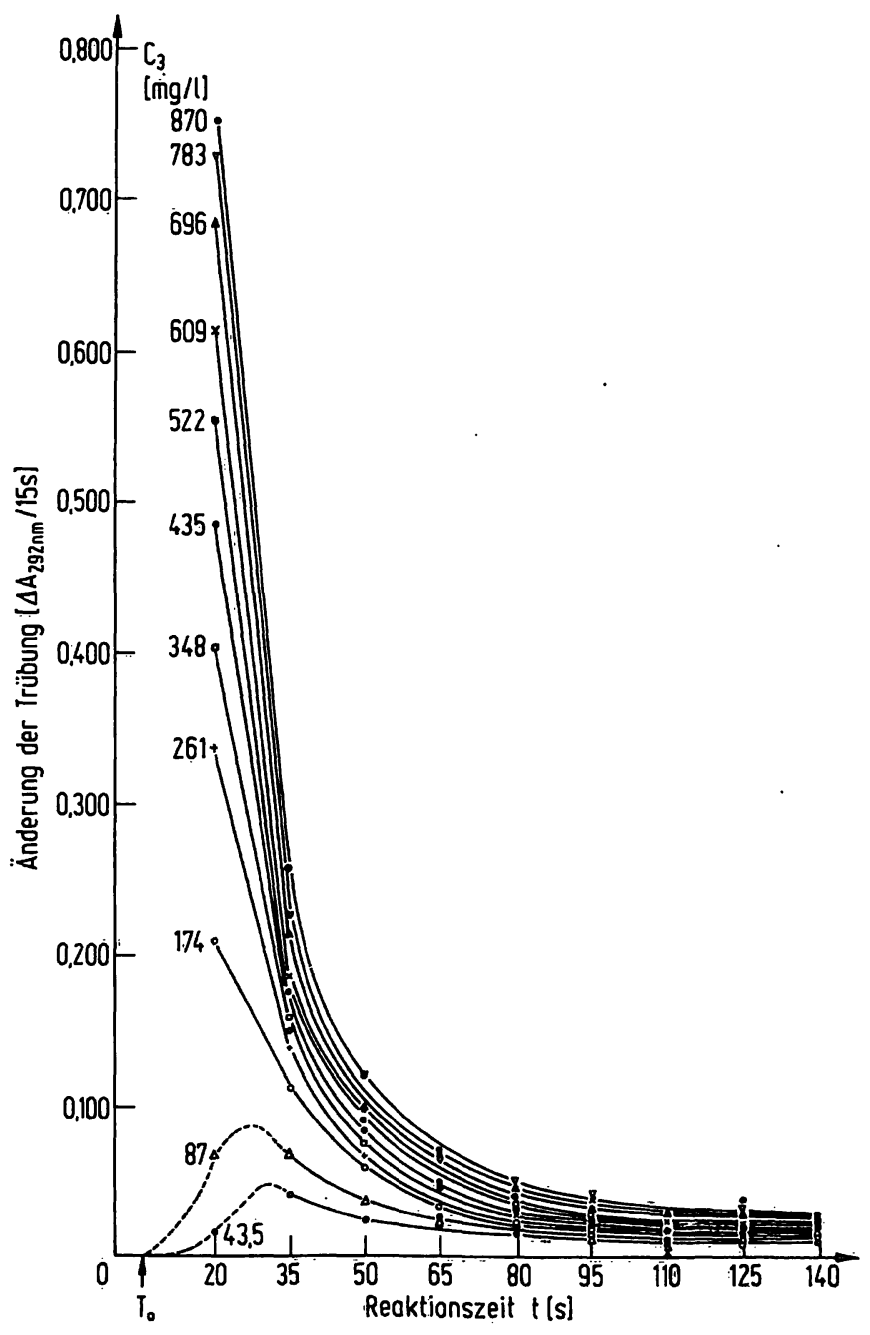

Abb. 1. Kinetische Trübungsänderung pro 15-Sekunden-Żeitintervall bei unterschiedlichen C3-Konzentrationen in Abhängigkeit von der Reaktionsżeit:

Antiserum-Vorverdünnungen $(1: 10)$ und Standard-HumanSerum-Vorverdünnungen mit Britton-Robinson-Puffer-NaCl-Lösung plus Nètżmittelzusatz, pH 6,00, $11 \mathrm{mmol} / \mathrm{1}$ Gesamtsalzkionzentration. 


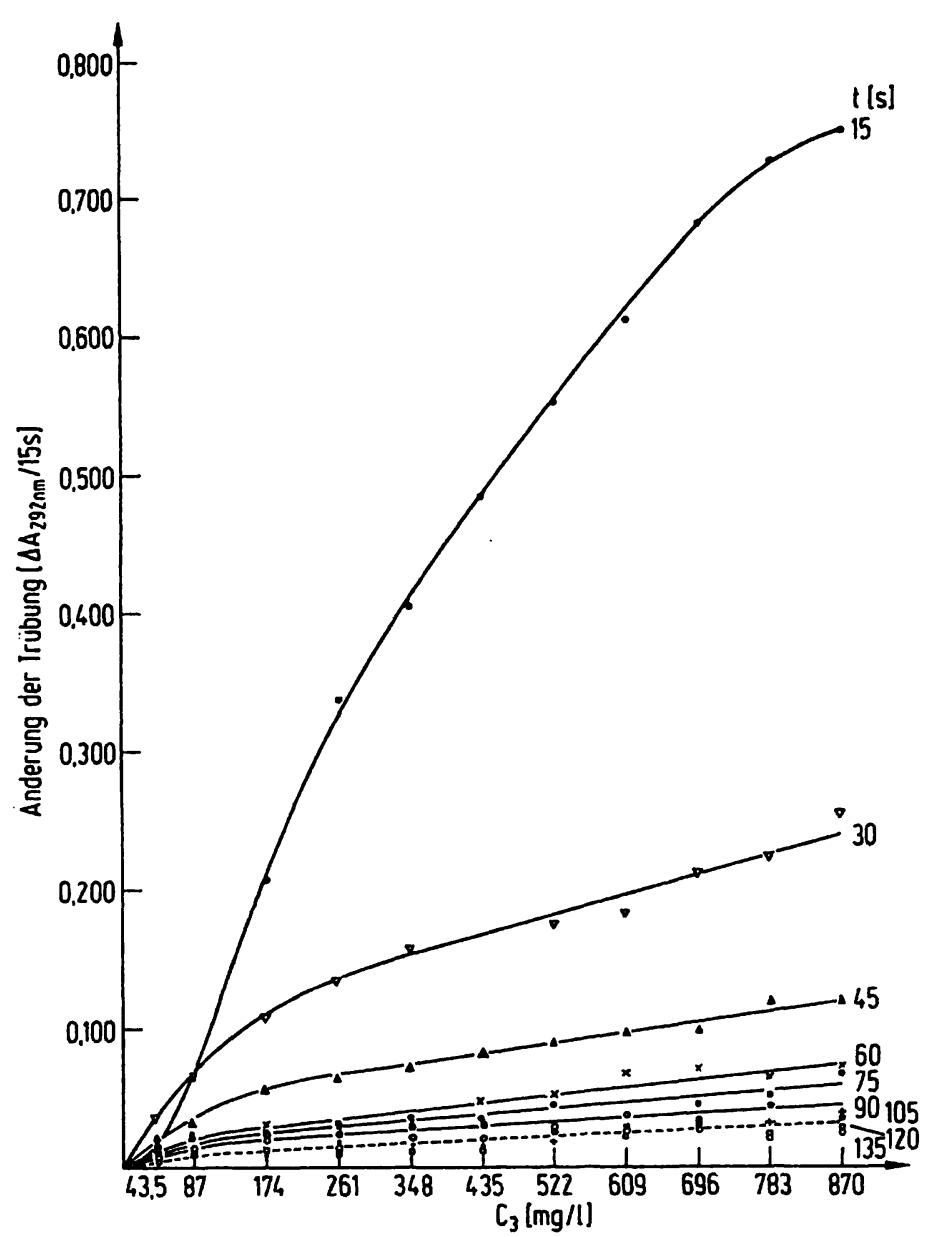

Abb. 2. Kinetische Trübungsänderung pro 15-Sekunden-Zeitintervall nach unterschiedlich langen Reaktionszeiten in Abhängigkeit von der C3-Konzentration:

Reaktionsbedingungen wie bei Abb. 1.

Wie die Abbildung 2 zeigt, lassen sich im Allgemeinen deutliche Unterschiede in der Trübungsänderung pro Zeitintervall $(\Delta \mathrm{A} / \Delta \mathrm{t})$ bei unterschiedlichen C3-Konzentrationen nur durch das Aufzeichnen von AnfangsKinetiken feststellen. So sind z. B. Reaktionszeiten von mehr als 60 Sekunden bei diesem System nicht mehr $\mathrm{zu}$ quantitativen Bestimmungen zu gebrauchen. Mit zunehmender Reaktionszeit nimmt nämlich die Trübungsänderung pro Zeitintervall rapide $\mathrm{ab}$ (s. auch $\mathrm{Abb} .1$ und 2).

Aus den Abbildungen 3 und 4 ist ersichtlich, daß kinetische immuno-turbidimetrische C3-Bestimmungen ebenfalls den Gesetzen der „Antikörper-Bestimmungskurven" (2) und ,Heidelberger-Kurven" gehorchen. Abbildung 3 zeigt die Trübungsänderungen nach $15 \mathrm{Se}$ kunden Reaktionszeit in Abhängigkeit von den jewei= ligen Antigen-Antikörpèr-Konżentrationen. Entsprechend werden niedrigere Antigen-Konzenträtionen mit höheren Antiserum:Vorverdünnungen und höhere Antigen-Konżentrationen mit niedrigeren AntiserumVorverdünnungen optimal bestimmt. Kleinere Antiserum-Vorverdünnungen als $1: 10$ führten hier bei höheren Antigen-Konzentrationen (äb etwa $170 \mathrm{mg} / \mathrm{l}$ ) aufgrund starker Präzipitat-Bildung und meßtechnisch bedingter, unerwünschter Sedimentation der Reaktionsprodukte durch das Zentrifugierprinzip zu falschen Meßergebnissen. Abbildung 4 dokumentiert, dạ sich

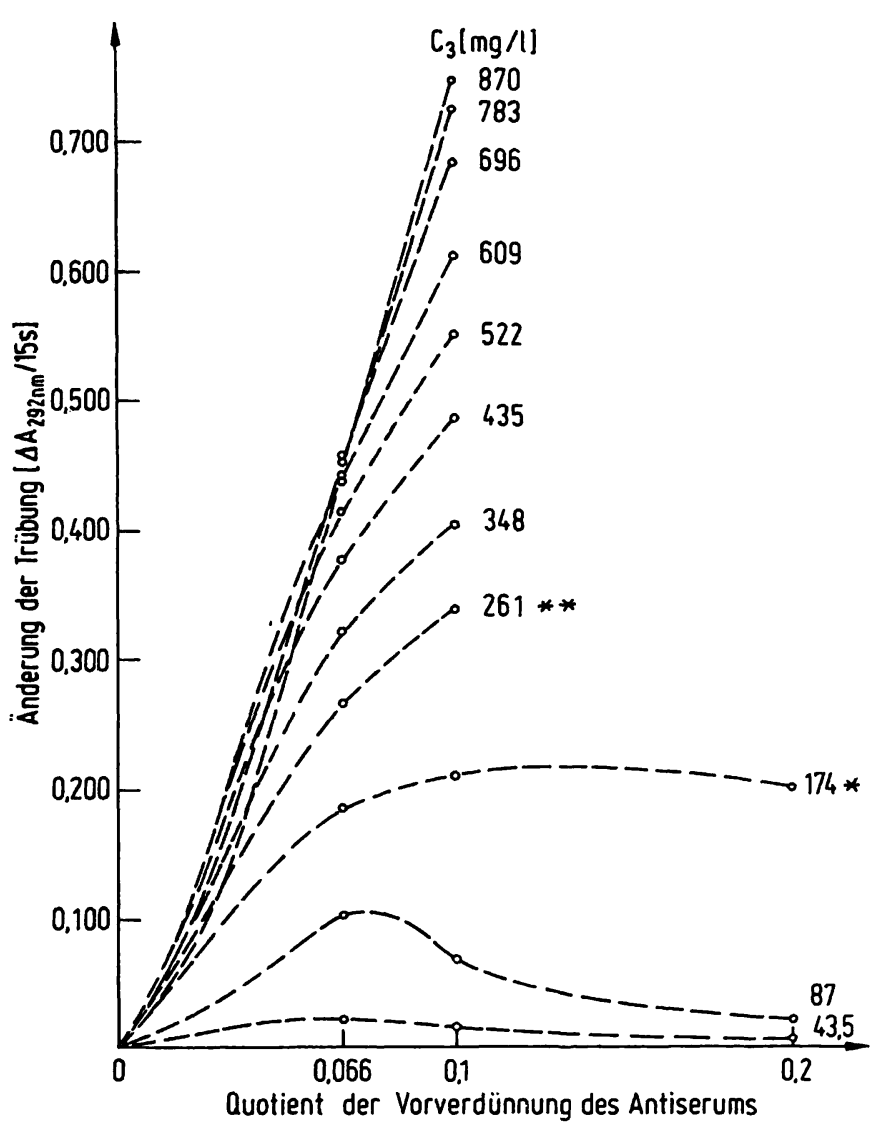

Abb. 3. Trübungsänderung nach 15 Sekunden Reaktionszeit bei unterschiedlichen C3-Konzentrationen in Abhängigkeit von der Antiserum-Konzentration:

$\star \rightarrow \star \star$ Zunehmende Sedimentierung der Präzipitate!

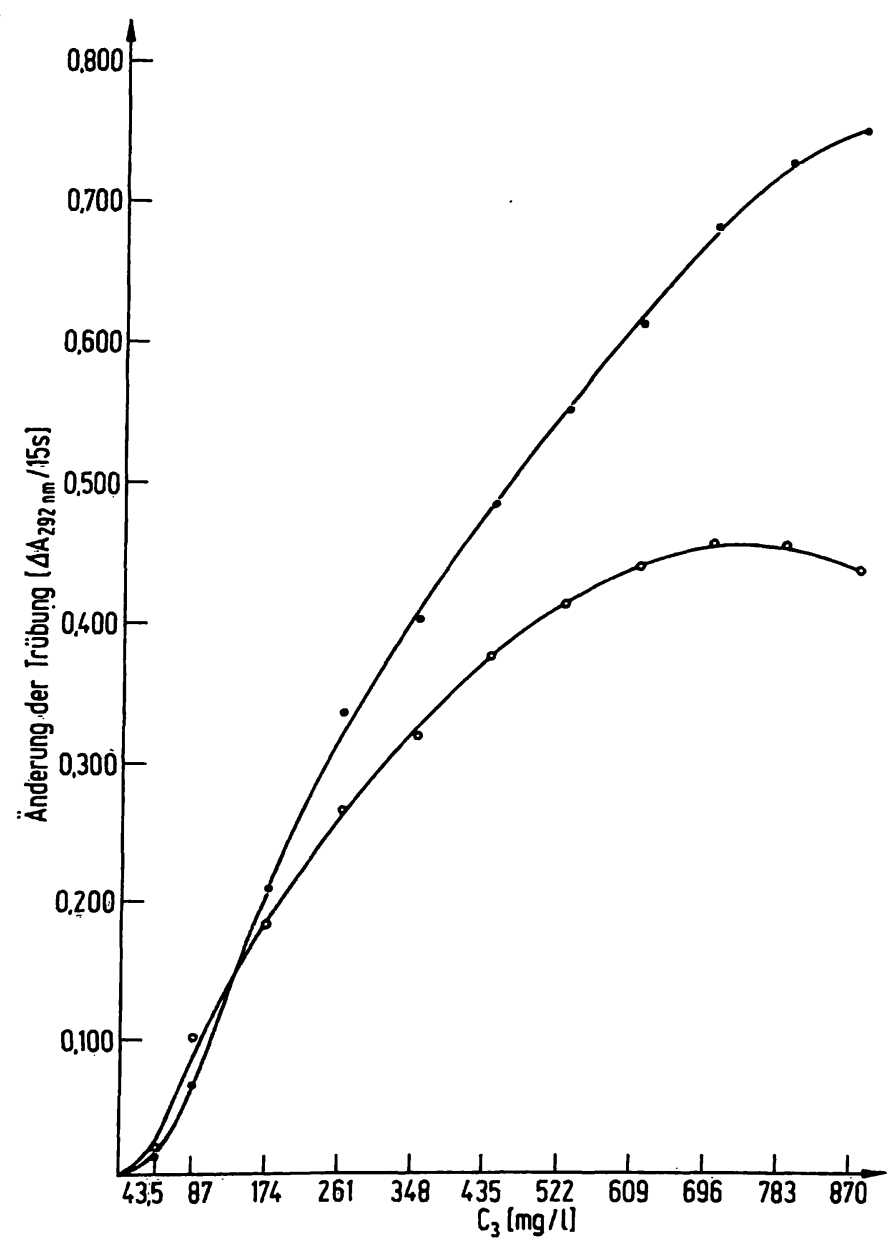

Abb. 4. Kinetisch-immuno-turbidimetrisch ermittelte „Heidelberger-Kurven":

- Antikörper-Vorverdünnung 1:10, 0 - $\circ$ Antikörper-Vorverdünnung 1:15. 


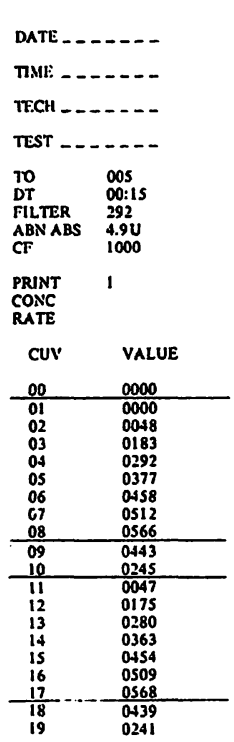

Abb. 5. C3-Standardkurve und Rechner-Ausdruck:

Werte kinetisch-immuno-turbidimetrisch mit Fast Analyzer CENTRIFICHEM SYSTEM 400 erhalten.

Werteausdruck:

CUV = Rotoreinsatz-Platznummern der Proben, VALUE $=\Delta \mathrm{A} / \Delta \mathrm{t}$.

C3-Standardwerte als Doppelbestimmung aus StandardHuman-Serum:

durch Messen der Anfangs-Kinetik in Abhängigkeit von der Antigen-Konzentration typische ,Heidelberger Kurven“ ergeben mit „Antikörper-Überschuß"-, „Äquivalenzpunkts"- und „Antigen-Überschuß"-Bereichen.

Abbildung 5 gibt ein C3-Standardkurven-Beispiel wieder, wie wir es unter den im Methodenteil dargestellten optimierten und standardisierten Bedingungen erhalten haben.

In Tabelle 1 und 2 sind relative Richtigkeit, Präzision pro Serie und von Tag zu Tag dargestellt und als zufriedenstellend zu bezeichnen.

Aus folgenden Gründen empfiehlt es sich, quantitative, kinetische immunologisch-turbidimetrische Einzelprotein-Analysen mit einem Zentrifugen-Fast-Analyzer durchzuführen:

Geringer Arbeitsaufwand, leichte Gerätebedienung, minimaler Reagenzien-Verbrauch (z. B. $1 \mathrm{ml}$ Antiserum für etwa 40 Analysen) und eine gute Qualitätskontrolle. Als besonderer Vorteil gegenüber A.I.P.-(= automatisierten Immunpräzipitations-) Methoden $(1,2)$ ist jedoch hervorzuheben, daß bei Messungen, die auf ,Initial-Rate". oder „Fixed-Time“-Kinetiken basieren, Bestimmungen der probenbedingten Eigentrübungen fortfallen (3-5). Eine zusätzliche beträchtliche Rationalisierung ergibt sich durch Einsatz eines Zentrifugen-Fast-Analyzers, da es mit diesem Gerät möglich ist, bereits schon nach 20 Sekunden in einem Meßdurchgang parallel 29 ProbenMeßwerte zu erhalten.
Tab. 1. Relative Richtigkeit von C3.

Überprüft mit Protein-Standard-Serum B:

Sollwert $=1230 \mathrm{mg} / \mathrm{l}$.

(Siehe auch Rechner-Ausdruck bei Abb. 5.)

\begin{tabular}{|c|c|}
\hline Platz-Nr. & Verdünnung \\
\hline $\begin{array}{l}09 / 18 \\
10 / 19\end{array}$ & $\begin{array}{l}1: 2,8 \\
1: 2 \text { von } 09 / 18\end{array}$ \\
\hline \multicolumn{2}{|c|}{ Ergebnis: $\begin{aligned} \bar{x} \text {-Istwert } & =1228,64 \mathrm{mg} / 1 \\
\mathrm{~s} & = \pm 46,28 \mathrm{mg} / 1 \\
\mathrm{VK} & =\quad 3,76 \% .\end{aligned}$} \\
\hline \multicolumn{2}{|c|}{$\begin{array}{l}\text { Abweichung: } \overline{\mathrm{x}} \text {-Istwert vom Sollwert }(=.100 \%) \text { : } \\
\begin{array}{ll}\text { absolut } & =-1,36 \mathrm{mg} / \mathrm{l} \\
\text { relativ } & =-0,11 \% .\end{array}\end{array}$} \\
\hline
\end{tabular}

Tab. 2. Qualitätskontrollen aus Standard-Human-Serum:

1. Präzision pro Serie, $N=20$ :

$$
\begin{aligned}
& \text { A } 87 \mathrm{mg} / \mathrm{l}: \\
& \overline{\mathrm{x}}=0,0894 \Delta \mathrm{A} / 15 \mathrm{~s}, \mathrm{~s}= \pm 0,0066, \mathrm{VK}=7,38 \% \\
& \text { B } 435 \mathrm{mg} / \mathrm{l}: \\
& \overline{\mathrm{x}}=0,5370 \Delta \mathrm{A} / 15 \mathrm{~s}, \mathrm{~s}= \pm 0,0074, \mathrm{VK}=1,38 \% \\
& \text { C } 783 \mathrm{mg} / \mathrm{l}: \\
& \overline{\mathrm{x}}=0,8061 \Delta \mathrm{A} / 15 \mathrm{~s}, \mathrm{~s}= \pm 0,0145, \mathrm{VK}=1,79 \%
\end{aligned}
$$

2. Präzision von Tag zu Tag, $N=10$ Tage:

$A \dot{\bar{x}}=217,5 \mathrm{mg} / \mathrm{l}, \mathrm{s}= \pm 9,78, \mathrm{VK}=4,30 \%$

- B $\overline{\mathrm{x}}=435,0 \mathrm{mg} / \mathrm{l}, \mathrm{s}= \pm 2 \overline{7}, 96, \mathrm{VK}=6,39 \%$

\section{Danksagung}

Frau Reckinger danke ich für sorgfältige technische Mitarbeit. 


\section{Literatur}

1. Killingsworth, L. M., Savory, J. \& Teague, P. O. (1971), Clin. Chem. 17, 374-377.

2. Ebeling, H. (1973), diese Z. 11, 209-214.

3. Tiffany, T. O., Parella, J. M., Johnson, W. F. \& Burtis, C. A. (1974), Clin. Chem. 20, 1055-1061.

4. Buffone, G. J., Cross, R. E., Savory, J. \& Soodak, C. (1974), Anal. Chem. 46, 2047-2049.
5. Buffone, G. J., Savory, J., Cross, R. E. \& Hammond, J. E. (1975), Clin. Chem. 21, 1731-1734.

6. Anderson, N. (1970), Amer. J. Clin. Pathol. 53, 778-785.

Dr. Herwig Ebeling

Institut für Klinische Chemie und Klinische Biochemie Klinikum Steglitz der Freien Universität Berlin Hindenburgdamm 30

D-1000 Berlin 45 
